Pacific Journal of Mathematics

ON SIMPLE ALGEBRAS OBTAINED FROM HOMOGENEOUS 


\title{
ON SIMPLE ALGEBRAS OBTAINED FROM HOMOGENEOUS GENERAL LIE TRIPLE SYSTEMS
}

\author{
Arthur A. SAgle
}

We continue the investigation of the simple anti-commutative algebras obtained from a homogeneous general L.t.s. In particular we consider the algebra which satisfies

$$
J(x, y, z) w=J(w, x, y z)+J(w, y, z x)+J(w, z, x y) .
$$

The usual process of analyzing a nonassociative algebra is to decompose it relative to elements whose right and left multiplications are diagonalizable linear transformations e.g. idempotents or Cartan subalgebras. In this paper we show that such a process yields only Lie algebras and indicates the difficulty in finding any non-Lie multiplication table for a simple anticommutative algebra satisfying (1).

A general Lie triple system [2] is an extension of a Lie triple system used in differential geometry and Jordan algebras. A general L.t.s. may be regarded as an anti-commutative algebra $A$ with a trilinear operation $[x, y, z]$ so that the mappings $D(x, y): z \rightarrow[x, y, z]$ are derivations of $A$ which generate a Lie algebra, $I(A)$, under commutation satisfying certain natural identities. A homogeneous general L.t.s. is a general L.t.s. for which the operation $[x, y, z]$ is a homogeneous expression in the products of $x, y$ and $z$; that is, using anticommutativity, $[x, y, z]=\alpha x y \cdot z+\beta y z \cdot x+\gamma z x \cdot y$ for some fixed $\alpha, \beta, \gamma$ in the base field. From [1] we see that if $A$ is a homogeneous general L.t.s. over a field of characteristic zero which is either an irreducible general L.t.s. or $I(A)$-irreducible or a simple algebra, then $A$ is a Lie or Malcev algebra or satisfies

$$
J(x, y, z) w=J(w, x, y z)+J(w, y, z x)+J(w, z, x y)
$$

where $J(x, y, z)=x y \cdot z+y z \cdot x+z x \cdot y$. The main result of this paper is the following theorem.

THEOREM. If $A$ is a simple finite dimensional anti-commutative algebra over a field $F$ of characteristic zero which satisfies (1) and if $A$ contains a nonzero element $u$ so that right multiplication by $u, R_{u}$, is a diagonalizable linear transformation, then $A$ is a Lie algebra.

Received August 3, 1964. Sponsored in part by NSF Grant GP-1453. 
2. Proof of theorem. For any anti-commutative algebra we have the identity

$$
\begin{gathered}
w J(x, y, z)-x J(y, z, w)+y J(z, w, x)-z J(w, x, y) \\
=J(w, x, y z)+J(w, y, z x)+J(w, z, x y) \\
+J(w x, y, z)+J(w y, z, x)+J(w z, x, y) .
\end{gathered}
$$

But using (1) we also have

$$
\begin{array}{r}
w J(x, y, z)-x J(y, z, w)+y J(z, w, x)-z J(w, x, y) \\
=-2[J(w, x, y z)+J(w, y, z x)+J(w, z, x y) \\
+J(w x, y, z)+J(w y, z, x)+J(w z, x, y)] .
\end{array}
$$

Thus using the two preceding identities we have

$$
\begin{aligned}
& J(w, x y, z)+J(w, y z, x)+J(w, z x, y) \\
& \quad=J(w x, y, z)+J(w y, z, x)+J(w z, x, y) .
\end{aligned}
$$

Now let $u \neq 0$ be an element of $A$ so that $R_{u}: x \rightarrow x u$ is a diagonalizable linear transformation. Then $R_{u} \neq 0$, for this implies that the one dimensional subspace $u F$ is an ideal of $A$ and therefore equals $A$. Thus $A^{2}=0$, a contradiction to the simplicity of $A$. Since $R_{u}$ acts diagonally in $A$ we may write

$$
A=A_{0} \oplus \sum_{\alpha \neq 0} A_{\alpha}
$$

where

$$
A_{\lambda}=\left\{x \in A: x\left(R_{u}-\lambda I\right)=0\right\} .
$$

We shall now prove

$$
A_{\alpha} A_{\beta} \subset A_{\alpha+\beta} \text {. }
$$

For let $x \in A_{\alpha}, y \in A_{\beta}$, then from (1)

$$
\begin{aligned}
J(u, x, y) R_{u} & =J(u, u, x y)+J(u, x, y u)+J(u, y, u x) \\
& =\beta J(u, x, y)-\alpha J(u, y, x) \\
& =(\alpha+\beta) J(u, x, y) .
\end{aligned}
$$

Thus $J(u, x, y) \in A_{\alpha+\beta}$ and therefore

$$
x y\left(R_{u}-(\alpha+\beta) I\right)=x y \cdot u+y u \cdot x+u x \cdot y \in A_{\alpha+\beta} .
$$

From this $x y\left(R_{u}-(\alpha+\beta) I\right)^{2}=0$ and setting $x y=\Sigma z_{\gamma} \in A_{0} \oplus \sum_{\alpha \neq 0} A_{\alpha}$ we see by the diagonal action of $R_{u}$ that $x y \in A_{\alpha+\beta}$. In particular (3) shows $A_{0}$ is a subalgebra of $A$.

Next we shall show 


$$
J\left(A_{\alpha}, A_{\beta}, A_{\gamma}\right)=0 \quad \text { or } \quad \alpha+\beta+\gamma=0
$$

for any characteristic roots $\alpha, \beta, \gamma$ of $R_{u}$. Let $x \in A_{\alpha}, y \in A_{\beta}, z \in A_{\gamma}$, then from (3) $J(x, y, z) \in A_{\alpha+\beta+\gamma}$ and therefore

$$
\begin{aligned}
(\alpha+\beta+\gamma) J(x, y, z)= & J(x, y, z) R_{u} \\
= & J(u, x, y z)+J(u, y, z x)+J(u, z, x y) \\
= & -\alpha x \cdot y z+(\alpha+\beta+\gamma) x \cdot y z+(\beta+\gamma) y z \cdot x \\
& -\beta y \cdot z x+(\alpha+\beta+\gamma) y \cdot z x+(\alpha+\gamma) z x \cdot y \\
& -\gamma z \cdot x y+(\alpha+\beta+\gamma) z \cdot x y+(\alpha+\beta) x y \cdot z \\
= & 0 .
\end{aligned}
$$

and this equation proves (4).

from (1) and (3) we have

$$
J\left(A_{0}, A_{0}, A_{0}\right) A_{0} \subset J\left(A_{0}, A_{0}, A_{0}\right)
$$

and for $\alpha \neq 0$ we have from (1), (3) and (4),

$$
\begin{aligned}
J\left(A_{0}, A_{0}, A_{0}\right) A_{\alpha} & \subset J\left(A_{\alpha}, A_{0}, A_{0}\right) \\
& =0 .
\end{aligned}
$$

Thus $J\left(A_{0}, A_{0}, A_{0}\right) A \subset J\left(A_{0}, A_{0}, A_{0}\right)$ and therefore $J\left(A_{0}, A_{0}, A_{0}\right)$ is an ideal of $A$ whicn is contained in $A_{0} \neq A$. Since $A$ is a simple algebra this yields

$$
J\left(A_{0}, A_{0}, A_{0}\right)=0 .
$$

Next we shall prove that if $\alpha$ is a nonzero characteristic root so that $-\alpha$ is also a characteristic root, then

$$
J\left(A_{\alpha}, A_{-\alpha}, A_{0}\right)=0 .
$$

For using (1), (3) and (5) we obtain

$$
J\left(A_{\alpha}, A_{-\alpha}, A_{0}\right) A_{0} \subset J\left(A_{\alpha}, A_{-\alpha}, A_{0}\right)
$$

and for any $\beta \neq 0$ we also obtain

$$
\begin{aligned}
J\left(A_{\alpha}, A_{-\alpha}, A_{0}\right) A_{\beta} \subset & J\left(A_{\beta}, A_{\alpha}, A_{-\alpha} A_{0}\right) \\
& +J\left(A_{\beta}, A_{-\alpha}, A_{0} A_{\alpha}\right) \\
& +J\left(A_{\beta}, A_{0}, A_{\alpha} A_{-\alpha}\right) \\
\subset & J\left(A_{\beta}, A_{\alpha}, A_{-\alpha}\right)+J\left(A_{\beta}, A_{0}, A_{0}\right) \\
= & 0,
\end{aligned}
$$

also using (4). Thus as in the proof of (5), $J\left(A_{\alpha}, A_{-\alpha}, A_{0}\right)$ is an ideal of $A$ which must be zero. Adopting the usual convention that if $\alpha$ is a characteristic root but $-\alpha$ is not, then $A_{-\alpha}=0$ we see that (6) holds 
for any characteristic root $\alpha$.

Next let

$$
B=\sum_{\alpha \neq 0} A_{\alpha} A_{-\alpha} \oplus \sum_{\alpha \neq 0} A_{\alpha},
$$

then if $\beta \neq 0$ we see from (3) that $B A_{\beta} \subset B$. If $\beta=0$, then from (6) we obtain $\left(A_{\alpha} A_{-\alpha}\right) A_{0} \subset A_{\alpha} A_{-\alpha}$ and therefore $B A_{0} \subset B$. Thus $B$ is an ideal of $A$ and therefore $B=0$ or $B=A$. If $B=0$, then $R_{u}=0$, a contradiction. Therefore we have

$$
A=\sum_{\alpha \neq 0} A_{\alpha} A_{-\alpha} \oplus \sum_{\alpha \neq 0} A_{\alpha}
$$

Now from (4) and (6) we have for any characteristic roots $\beta$ and $\alpha \neq 0$, $J\left(A_{\alpha}, A_{-\alpha}, A_{\beta}\right)=0$ and therefore

$$
J\left(A_{\alpha}, A_{-\alpha}, A\right)=0 \quad(\alpha \neq 0) .
$$

We shall use (7) and (8) together with the following lemma to prove $A$ is a Lie algebra.

Lemma. Let $N=\{x \in A: J(x, A, A)=0\}$, then

(i) $J(a, b, A)=0$ implies $a b \in N$;

(ii) $N$ is an ideal of $A$ which is a Lie algebra.

Proof. Clearly (ii) follows from (i). So let $a, b \in A$ be such that $J(a, b, A)=0$ and let $w, z \in A$. Then from (1) and (2) we have

$$
\begin{aligned}
0 & =w J(a, b, z) \\
& =J(w, a b, z)+J(w, b z, a)+J(w, z a, b) \\
& =J(w a, b, z)+J(w b, z, a), \text { using }(2) .
\end{aligned}
$$

Now interchanging $z$ and $w$ in this last equation we obtain $0=$ $J(z a, b, w)+J(z b, w, a)=J(w, b z, a)+J(w, z a, b)$ and using this in (9) yields $J(a b, w, z)=0$; that is, $a b \in N$.

To show that $A$ is a Lie algebra, suppose it is not. Then from the lemma $N=0$ and from (8) $A_{\alpha} A_{-\alpha} \subset N=0$. Thus from (7) $A=$ $\sum_{\alpha \neq 0} A_{\alpha}$ and therefore $A_{0}=0$; this contradicts $0 \neq u \in A_{0}$.

\section{BIBLIOGRAPHY}

1. A. Sagle, On anti-commutative algebras and general Lie triple systems, to appear in Pacific J. Math.

2. K. Yamaguti, On the Lie triple system and its generalization, J. Sci. Hiroshima University, 21 (1958), 155-160. 


\title{
PACIFIC JOURNAL OF MATHEMATICS
}

\author{
EDITORS
}

\author{
H. SAmelson \\ Stanford University \\ Stanford, California \\ R. M. Blumenthal \\ University of Washington \\ Seattle, Washington 98105
}

\author{
J. DugundjI \\ University of Southern California \\ Los Angeles, California 90007
}

*Richard Arens

University of California

Los Angeles, California 90024

\section{ASSOCIATE EDITORS}
E. F. BECKENBACH
B. H. NeUmanN
F. WOLF
K. YoSIDA

\section{SUPPORTING INSTITUTIONS}

\author{
UNIVERSITY OF BRITISH COLUMBIA \\ CALIFORNIA INSTITUTE OF TECHNOLOGY \\ UNIVERSITY OF CALIFORNIA \\ MONTANA STATE UNIVERSITY \\ UNIVERSITY OF NEVADA \\ NEW MEXICO STATE UNIVERSITY \\ OREGON STATE UNIVERSITY \\ UNIVERSITY OF OREGON \\ OSAKA UNIVERSITY \\ UNIVERSITY OF SOUTHERN CALIFORNIA
}

\author{
STANFORD UNIVERSITY \\ UNIVERSITY OF TOKYO \\ UNIVERSITY OF UTAH \\ WASHINGTON STATE UNIVERSITY \\ UNIVERSITY OF WASHINGTON \\ * * * * \\ AMERICAN MATHEMATICAL SOCIETY \\ CALIFORNIA RESEARCH CORPORATION \\ SPACE TECHNOLOGY LABORATORIES \\ NAVAL ORDNANCE TEST STATION
}

Mathematical papers intended for publication in the Pacific Journal of Mathematics should by typewritten (double spaced). The first paragraph or two must be capable of being used separately as a synopsis of the entire paper. It should not contain references to the bibliography. No separate author's resumé is required. Manuscripts may be sent to any one of the four editors. All other communications to the editors should be addressed to the managing editor, Richard Arens, at the University of California, Los Angeles, California 90024.

50 reprints per author of each article are furnished free of charge; additional copies may be obtained at cost in multiples of 50 .

The Pacific Journal of Mathematics is published quarterly, in March, June, September, and December. Effective with Volume 13 the price per volume (4 numbers) is $\$ 18.00$; single issues, $\$ 5.00$. Special price for current issues to individual faculty members of supporting institutions and to individual members of the American Mathematical Society: $\$ 8.00$ per volume; single issues $\$ 2.50$. Back numbers are available.

Subscriptions, orders for back numbers, and changes of address should be sent to Pacific Journal of Mathematics, 103 Highland Boulevard, Berkeley 8, California.

Printed at Kokusai Bunken Insatsusha (International Academic Printing Co., Ltd.), No. 6, 2-chome, Fujimi-cho, Chiyoda-ku, Tokyo, Japan.

PUBLISHED BY PACIFIC JOURNAL OF MATHEMATICS, A NON-PROFIT CORPORATION

The Supporting Institutions listed above contribute to the cost of publication of this Journal, but they are not owners or publishers and have no responsibility for its content or policies.

* Basil Gordon, Acting Managing Editor until February 1, 1966. 


\section{Pacific Journal of Mathematics}

\section{Vol. 15, No. $4 \quad$ December, 1965}

Robert James Blattner, Group extension representations and the structure space ........... 1101

Glen Eugene Bredon, On the continuous image of a singular chain complex .............. 1115

David Hilding Carlson, On real eigenvalues of complex matrices .................... 1119

Hsin Chu, Fixed points in a transformation group ............................. 1131

Howard Benton Curtis, Jr., The uniformizing function for certain simply connected Riemann

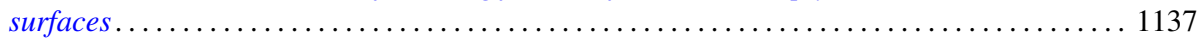

George Wesley Day, Free complete extensions of Boolean algebras................... 1145

Edward George Effros, The Borel space of von Neumann algebras on a separable Hilbert

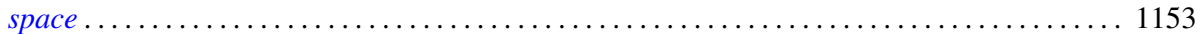

Michel Mendès France, $A$ set of nonnormal numbers ......................... 1165

Jack L. Goldberg, Polynomials orthogonal over a denumerable set ................ 1171

Frederick Paul Greenleaf, Norm decreasing homomorphisms of group algebras . . . . . . . . 1187

Fletcher Gross, The 2-length of a finite solvable group ........................ 1221

Kenneth Myron Hoffman and Arlan Bruce Ramsay, Algebras of bounded sequences ........ 1239

James Patrick Jans, Some aspects of torsion . . . . . . . . . . . . . . . . . . . . . . . 1249

Laura Ketchum Kodama, Boundary measures of analytic differentials and uniform

approximation on a Riemann surface ............................... 1261

Alan G. Konheim and Benjamin Weiss, Functions which operate on characteristic

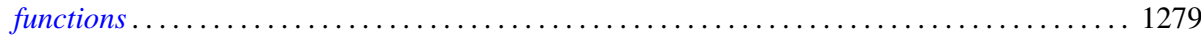

Ronald John Larsen, Almost invariant measures ............................ 1295

You-Feng Lin, Generalized character semigroups: The Schwarz decomposition ............ 1307

Justin Thomas Lloyd, Representations of lattice-ordered groups having a basis . . . . . . . . 1313

Thomas Graham McLaughlin, On relative coimmunity ....................... 1319

Mitsuru Nakai, $\Phi$-bounded harmonic functions and classification of Riemann surfaces ....... 1329

L. G. Novoa, On n-ordered sets and order completeness ..................... 1337

Fredos Papangelou, Some considerations on convergence in abelian lattice-groups . . . . . . . 1347

Frank Albert Raymond, Some remarks on the coefficients used in the theory of homology

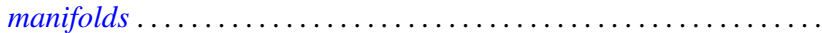

John R. Ringrose, On sub-algebras of a $C^{*}$-algebra .

Jack Max Robertson, Some topological properties of certain spaces of differentiable

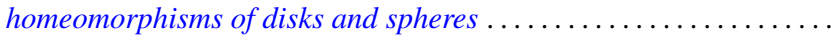

Zalman Rubinstein, Some results in the location of zeros of polynomials

Arthur Argyle Sagle, On simple algebras obtained from homogeneous general Lie triple systems. . . .

Hans Samelson, On small maps of manifolds ............................... 1401

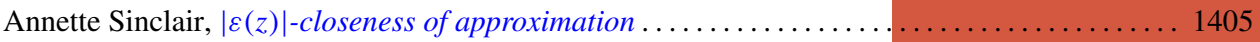

Edsel Ford Stiel, Isometric immersions of manifolds of nonnegative constant sectional curvature

Earl J. Taft, Invariant splitting in Jordan and alternative algebras ................. 1421

L. E. Ward, On a conjecture of R. J. Koch . . . . . . . . . . . . . . . . . . . . . . . . . . . 1429

Neil Marchand Wigley, Development of the mapping function at a corner . . . . . . . . . . 1435

Horace C. Wiser, Embedding a circle of trees in the plane ....................... 1463

Adil Mohamed Yaqub, Ring-logics and residue class rings . . . . . . . . . . . . . . . . 1465

John W. Lamperti and Patrick Colonel Suppes, Correction to: Chains of infinite order and their application to learning theory ........................................ 1471

Charles Vernon Coffman, Correction to: Non-linear differential equations on cones in Banach

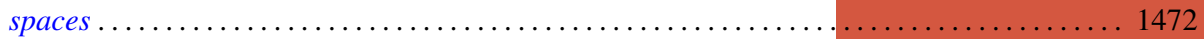

P. H. Doyle, III, Correction to: A sufficient condition that an $\operatorname{arc}$ in $S^{n}$ be cellular. . . . . . . . 1474

P. P. Saworotnow, Correction to: On continuity of multiplication in a complemented algebra 